\title{
Hyperspectral Imaging Under Low Illumination with a Single Photon Camera
}

\author{
Alexander D. Griffiths \\ Institute of Photonics \\ University of Strathclyde \\ Robert K. Henderson \\ University of Edinburgh \\ Edinburgh, UK \\ robert.henderson@ed.ac.uk
}

Glasgow, UK Glasgow, UK

Haochang Chen

SIPBS

University of Strathclyde

Glasgow, UK

\author{
Michael J. Strain \\ Institute of Photonics \\ University of Strathclyde \\ Glasgow, UK \\ michael.strain@strath.ac.uk
}

\author{
Johannes Herrnsdorf \\ Institute of Photonics \\ University of Strathclyde \\ Glasgow, UK
}

\author{
David Li \\ SIPBS \\ University of Strathclyde \\ Glasgow, UK \\ david.li@strath.ac.uk
}

\author{
Martin D. Dawson \\ Institute of Photonics \\ University of Strathclyde \\ Glasgow, UK \\ m.dawson@strath.ac.uk
}

\begin{abstract}
Conventional sensors for hyperspectral imaging are limited by noise when operated in low illumination conditions. Recent advances in single-photon avalanche diode (SPAD) arrays provide image sensors capable of single photon counting imaging. Such devices provides camera systems which can operate under low illumination levels and high frame rates, with fully digital in-pixel processing capabilities. Here, we present a hyperspectral imaging system based on low level illumination of a target scene using nine LEDs, each with different emission wavelengths. The backscattered light is captured by a SPAD camera, which can recover spectral information based on the number of photon counts received for each illumination wavelength.
\end{abstract}

Index Terms-LED, SPAD, Hyperspectral imaging

\section{INTRODUCTION}

Hyperspectral imaging, the measurement of spectral information across a 2D spatial image, has been used in remote sensing applications for many years [1]. The additional spectral information aids the identification of features in an image, as materials reflect, absorb or emit electromagnetic radiation in different ways, depending on their composition and structure. More recently, multiwavelength imaging has proved beneficial in food quality analysis [2], medical studies [3], and analysis of artistic and historical objects [4].

Typically, hyperspectral imaging systems use an image sensor with either dispersive optics or narrow band filters to discriminate between incident wavelengths [5]. In the dispersive case, light is collected through a collimating slit and spread across the sensor by a diffraction grating or prism. One line of the image is captured, as the second dimension of the image sensor is used

This work was funded by the UK Engineering and Physical Sciences Research Council (EPSRC) Quantum Hub in Quantum Enhanced Imaging (EP/M01326X/1). The underlying datasets are available at http://dx.doi.org/10.15129/3de27021-520e-4e1b-9501-c5d95360af2b to determine spectral information. The camera or target must therefore be moved to scan lines sequentially to build up a full hyperspectral image. Alternatively, full images can be taken through a set of beam-splitting prisms with a series of image sensors, though this rapidly becomes impractical with increasing number of wavelengths. In the filtered case, a series of images is taken sequentially with different narrow band filters in front of an image sensor. As the number of bands increases, so does the length of time to record a full hyperspectral image as the filters must be changed. This restricts the frame rate of a camera system, limiting its ability to capture high speed events. Multiple filters can be also be arranged directly on the pixels of an image sensor, similar to conventional colour imaging [6]. However, this reduces the image resolution as pixels are divided up between many wavelengths.

For hyperspectral imaging, CCD or CMOS image sensors are often used. While these devices can have high detection efficiency, single-photon resolution is difficult due to noise, and usually performed using photo-cathode image intensification techniques [7]. These systems have a number of practical disadvantages, such as high cost, high voltages and vacuum requirements. Single-photon avalanche diodes (SPADs) offer an alternative technology for high performance image sensors [8]. These photodetectors are solid state, operate in the visible range, and are capable of detecting single-photons with high timing resolution. By arranging such devices in two dimensions, camera systems with single-photon sensitivity and digital in-pixel processing capabilities are possible. Furthermore, timing electronics can be implemented to enable time-correlated single-photon counting (TCPSC) measurements to be performed on a single chip [9]. Such camera systems have found use in fluorescence lifetime imaging [10], 3-D ranging [11] and light-in- 
flight imaging [12].

Here, we present a system for hyperspectral imaging based on LED illumination of a target scene. A SPAD camera system [13] is used to capture shortexposure, sequential images illuminated by LEDs with different emission wavelengths. Spectral information can be determined based on the number of photon counts received for each illumination condition. The LEDs are supplied with a low driving current, providing low levels of illumination. The high frame rate of the SPAD camera potentially allows hyperspectral data to be collected at kilo-frames-per-second rates at the full resolution of the image sensor.

\section{Methods}

\section{A. SPAD Camera \& LED Array}

The SPAD image sensor used is a $3.15 \mathrm{~mm} \times 2.37 \mathrm{~mm}$ chip, fabricated in $40 \mathrm{~nm}$ CMOS technology, and consists of $192 \times 128$ pixels [13]. Each $18.4 \mu \mathrm{m} \times 9.2 \mu \mathrm{m}$ pixel has a $13 \%$ fill factor and contains the necessary circuitry to perform both timing and photon counting functions, though here we only make use of the photon counting mode. The SPAD pixels in the sensor have been optimised to minimise dark count rate (DCR) at room temperature, and maximise photon detection probability (PDP), while maintaining a low breakdown voltage [14]. The median DCR is $50 \mathrm{~Hz}$ at $24^{\circ} \mathrm{C}$, with PDP peaking at $33 \%$ for green wavelengths. A number of pixels in the array have significantly higher DCR than the median, causing bright spots in the captured images. These "hot pixels" were accounted for by taking a long exposure measurement in dark conditions so that the average number of counts in each pixel for a given exposure time could be subtracted in further measurements.

The camera chip is housed in a ceramic package on a printed circuit board (PCB), where chip control and signal readout is performed with a field programmable gate array (FPGA) (Opal Kelly XEM6310). A user interface in MATLAB ${ }^{\mathrm{TM}}$ allows control over the voltages, operational mode, and exposure times, as well as the acquisition of image frames. A set of camera optics (Navitar MVL8M23) were mounted on the SPAD camera, providing a field of view for the image sensor of approximately $15^{\circ}$.

Different illumination conditions were produced using an array of commercially available LEDs (Lumileds Luxeon $\mathrm{Z}$ series) mounted on a PCB with control electronics. The LEDs are $1 \mathrm{~mm}^{2}$ and arranged in a ring with a diameter of $1 \mathrm{~cm}$ to avoid variation in illumination due to position. No optics were used for light collection and the electrical supply to each LED is current-limited at $5 \mathrm{~mA}$. The peak emission wavelengths and output optical power for each LED at this driving current are shown in Table I. For the experimental results, the LED board was mounted next to the camera at a distance of $0.7 \mathrm{~m}$ from the target. Due to the wide beam angle of the LEDs, this results in broad, roughly uniform, low intensity illumination of the imaged scene. Table I also shows there is significant variation in the output optical power from each LED, due to variations in efficiency across different devices. In addition to the spectral efficiency of the SPAD, this must be accounted for during calibration.

TABLE I

EMISSION WAVELENGTH AND EMITTED OPTICAL POWER OF EACH LED WHEN DRIVEN AT 5 MA.

\begin{tabular}{ccc}
\hline Designation & Wavelength $(\mathrm{nm})$ & Optical power $(\mathrm{mW})$ \\
\hline Red & 630 & 1.21 \\
Red-orange & 615 & 1.11 \\
Amber & 590 & 0.43 \\
Green & 530 & 2.23 \\
Cyan & 500 & 2.43 \\
Blue & 470 & 3.84 \\
Royal blue & 450 & 4.42 \\
Violet & 420 & 3.40 \\
"Ultra violet" & 415 & 2.40 \\
\hline
\end{tabular}

Digital signals from an FPGA (Opal Kelly XEM6310) are used to control LED activation. The LED array is capable of switching the active element at high speeds, in excess of $1 \mathrm{MHz}$. However, for the experiments shown here the active element was switched at a slow rate, with images taken by manually triggering the SPAD camera. This gives an image for each illumination wavelength for offline processing. In a future system, the LED array could be triggered directly with an output from the SPAD camera, allowing each illumination condition to be imaged rapidly and fully utilise the high frame rate of the camera.

\section{B. Reflectance Calculation \& Calibration}

The spectral reflectance $R(\lambda)$ of a target is determined by:

$$
R(\lambda)=\frac{\sigma_{r}}{\sigma_{i}} .
$$

Where $\sigma_{i}$ is the incident spectra on the target and $\sigma_{r}$ is the spectra received by the camera. As both the illumination and reflected light is divergent, an absolute measurement of either spectra is very difficult. However, the relative emission spectrum from the transmitter can be accounted for, therefore relative measurements of reflectance spectra can be made by measuring the amount of light from each wavelength collected by the camera.

Both the illumination optical power and sensitivity of the SPAD are wavelength dependent, so the setup must be calibrated. A relative calibration factor is needed for each emission wavelength, which can then be used to adjust the photon counts for each pixel. This was determined experimentally by directly imaging the LED array with the SPAD camera and counting the number of photons received from each LED. The counted photons determine the relative amount of light detected with no reflectance target present. Their normalised values can then be used to scale reflectance count measurements. In 
effect, this is a determination of $\sigma_{i}$ in Equation 1, while $\sigma_{r}$ will be given by the measured photon counts on the SPAD array. The calibration factors are shown in Fig. 1. It can be seen that the system receives the highest photon counts in the blue and green regions of the spectrum, with reduced contributions in the red and violet. While the LED emitters are more efficient in blue region, the SPAD pixels have peak detection efficiency around $520 \mathrm{~nm}$, in the green [14]. The detection efficiency is dominant over the LED efficiency, producing the calibration curve shown.

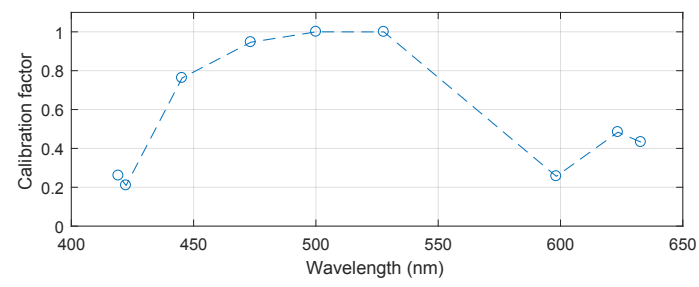

Fig. 1. Calibration factors for each illumination wavelength.

\section{RESUlTS \& DisCUSSION}

The target scene was the CIE 1931 colour space chromaticity diagram [15], printed on white paper. At a distance of $0.7 \mathrm{~m}$ the camera imaged an area approximately 19 by $14 \mathrm{~cm}$. The SPAD camera and LED transmitter board were arranged next to each other, such that the light from the LEDs illuminates a scene, and the SPAD camera collects the backscattered light. Data was taken with the laboratory lights switched off to minimise background counts. Fig. 2 shows single image frames under illumination from each LED. On average, each pixel in the array receives 5.59 counts due to the low illumination level and exposure time of $1 \mathrm{~ms}$. A conventional camera operating with a comparable exposure time records no visible image under these conditions, however, the SPAD camera is able to discern some degree of spatial information about the scene. Despite the strong Poissonian noise of the few-photon count images, it can also be seen that different regions of the chromaticity diagram reflect different amounts of light, dependent on the illumination wavelength. As the single frame images are strongly influenced by Poissonian noise, it is useful to consider the integration of multiple frames to produce a cleaner image. Fig. 3 shows the result of 100 frames integrated together. As expected, the results are very similar to the single frame case, but with significantly reduced levels of noise.

An approximation for a colour image of the scene can be made by combining three different illumination wavelength images in an RGB fashion. This was done using the red-orange, green and royal blue LEDs, as these are the closest matches to the peak wavelengths of the CIE colour matching functions. The resulting RGB

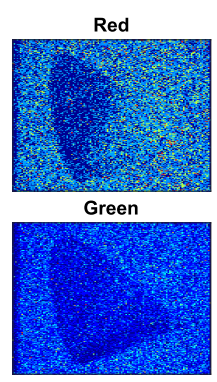

Royal blue
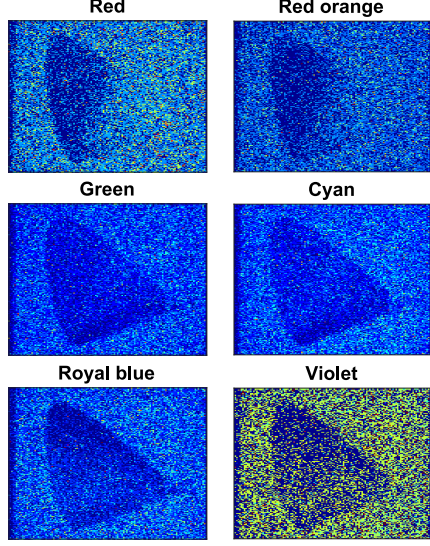

Cyan

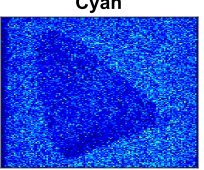

Violet

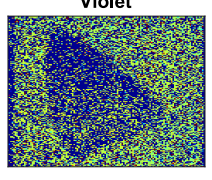

Fig. 2. Calibrated reflectance count levels for a single frame.
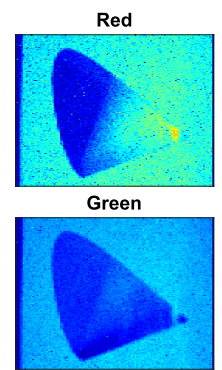

Royal blue
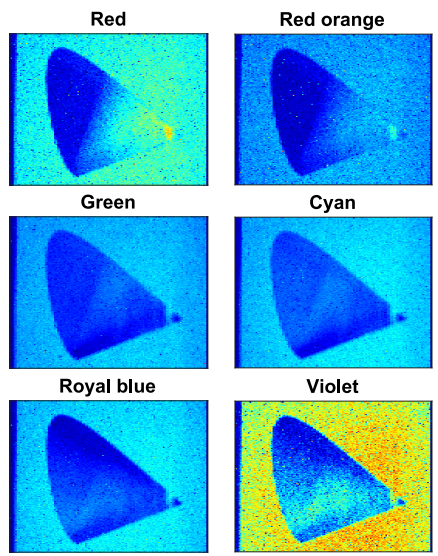

Cyan

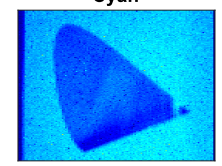

Violet
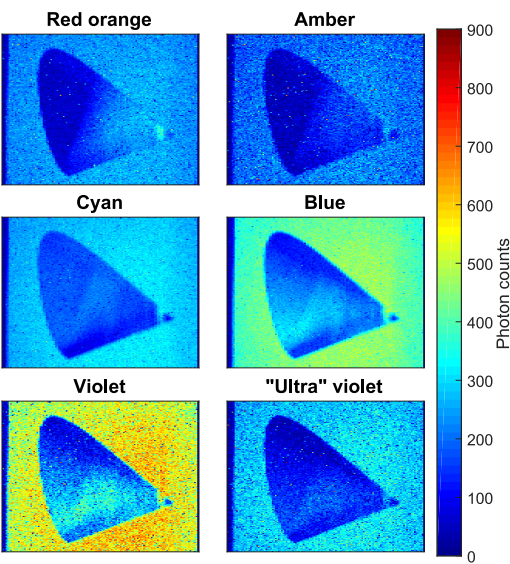

Fig. 3. Calibrated reflectance count levels for 100 integrated frames.

approximation images are shown in the left of Fig. 4 for both the single frame and integrated cases. Colour science is a complex field, and accurately reproducing images as seen by human vision is beyond the scope of this work, however, the RGB approximations serve as a useful means of visualising the scene. The single frame image shows strongly varying colours on a pixelby-pixel level, due to the Poissonian noise. However, the target image can still be resolved, and different coloured regions identified. The integrated image smooths out the random fluctuations, giving a cleaner image with more consistent pixel colours across each region.

Fig. 4 also shows relative reflectance spectra for six regions across the image, indicated in white on the integrated RGB image (i-vi). These spectra are determined by averaging a $5 \times 5$ region of pixels in the images, to account for the significant variations in the single frame case. While it is difficult to make a quantitative comparison to actual reflectance spectra, each plot shows the general trends expected for the colours present, 


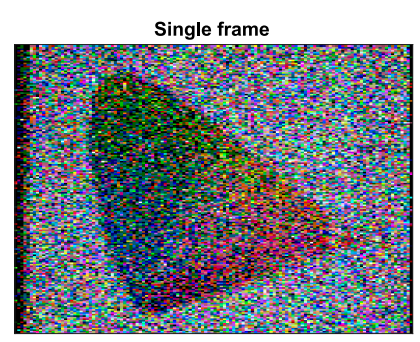

100 integrated frames
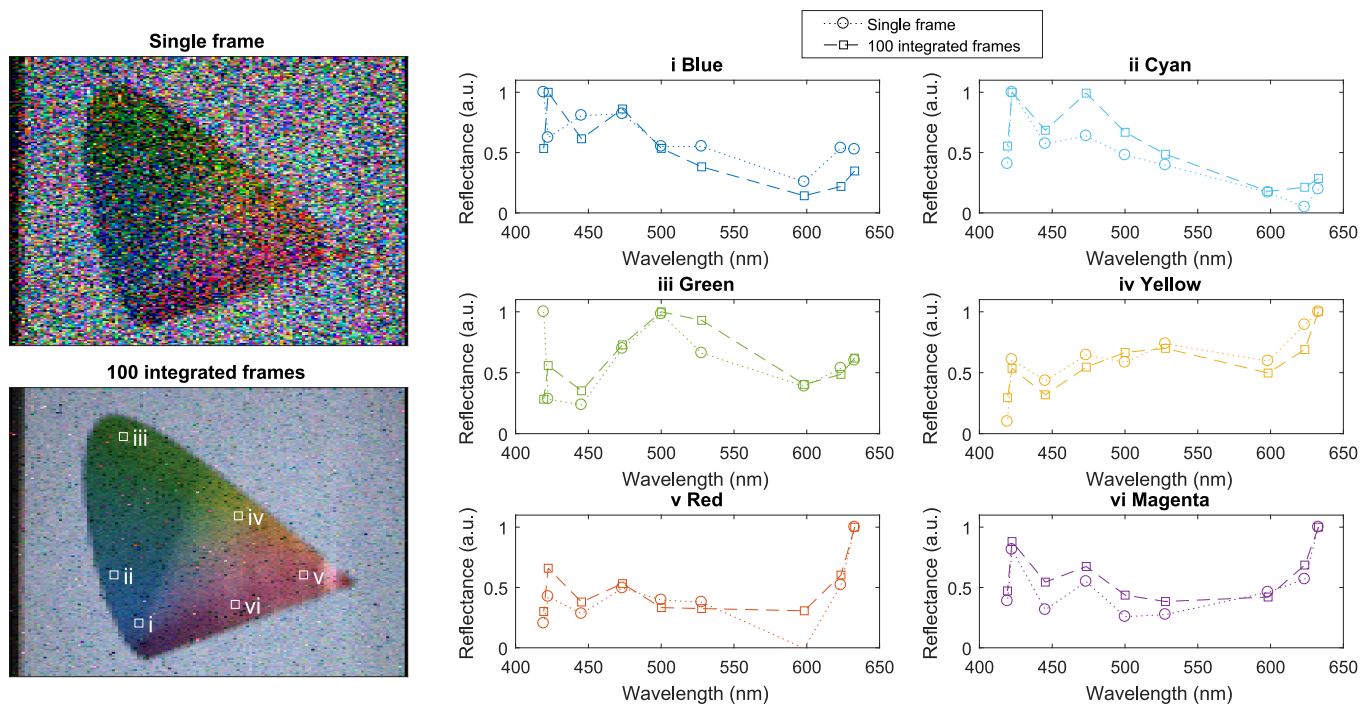

Fig. 4. Spectral reflectance of different coloured regions of the CIE diagram.

and could therefore be used to provide some level of discrimination between features. Notably, the single frame dataset provides similar reflectance information to the integrated case. While this will partially be due to the averaging over a region of the image, it demonstrates that potentially useful spectral information can be captured with a very low number of photon counts and frames.

The current configuration uses an exposure time of $1 \mathrm{~ms}$ for each of the 9 wavelengths. With suitable synchronisation electronics, hyperspectral images could be acquired under these conditions at $111 \mathrm{fps}$. The sensors maximum frame rate of $18.6 \mathrm{kfps}$ [13] would allow hyperspectral images to be taken at a frame rate of $2.07 \mathrm{kfps}$. This technique therefore allows high frame rate hyperspectral imaging to be performed in low illumination environments with the full resolution of the image sensor.

\section{Conclusions}

Hyperspectral imaging has been demonstrated using a SPAD camera and a set of LEDs for varying illumination wavelengths. Proof-of-concept images have been shown, with identifiably different spectra received from different regions of a printed chromaticity diagram. By changing illumination conditions, rather than filtering or using dispersive optics, the full resolution of the image sensor is used and the high frame-rate performance can be preserved. This technique can easily be adjusted to measure other wavelength bands, provided they fall within the spectral response of the SPADs, and LEDs can be produced. Even with few detected photons, the SPAD camera produces images with identifiable spectral features.

\section{REFERENCES}

[1] A. F. Goetz, G. Vane, J. E. Solomon, and B. N. Rock, "Imaging spectrometry for Earth remote sensing.," Science, vol. 228, no. 4704, pp. 1147-53, 1985.

[2] K. Sendin, P. J. Williams, and M. Manley, "Near infrared hyperspectral imaging in quality and safety evaluation of cereals," Critical Reviews in Food Science and Nutrition, vol. 58, no. 4 , pp. 575-590, 2018.

[3] G. Lu and B. Fei, "Medical hyperspectral imaging: a review," Journal of Biomedical Optics, vol. 19, no. 1, p. 010901, 2014.

[4] C. Balas et al., "A novel hyper-spectral imaging apparatus for the non-destructive analysis of objects of artistic and historic value," Journal of Cultural Heritage, vol. 4, pp. 330-337, 2003.

[5] A. Robles-Kelly and C. Huynh, Imaging Spectroscopy for Scene Analysis. London: Springer, 2013.

[6] B. Geelen, N. Tack, and A. Lambrechts, "A compact snapshot multispectral imager with a monolithically integrated per-pixel filter mosaic," Proc. SPIE, vol. 8974, p. 8974, 2014.

[7] P. Seitz and A. Theuwissen, Singe-Photon Imaging. London: Springer, 2011.

[8] E. Charbon, "Single-photon imaging in complementary metal oxide semiconductor processes," Philosophical Transactions of the Royal Society A, vol. 372, no. 2012, 2014.

[9] J. Richardson et al., "A $32 \times 3250$ ps resolution 10 bit time to digital converter array in 130nm CMOS for time correlated imaging," Proceedings of the Custom Integrated Circuits Conference, no. 029217, pp. 77-80, 2009.

[10] D.-U. Li et al., "Real-time fluorescence lifetime imaging system with a $32 \times 320.13 \mu \mathrm{m}$ CMOS low dark-count single-photon avalanche diode array," Optics Express, vol. 18, no. 10, p. 10257, 2010.

[11] D. Bronzi et al., "100 000 frames/s $64 \times 32$ single-photon detector array for 2-D imaging and 3-D ranging," IEEE Journal on Selected Topics in Quantum Electronics, vol. 20, no. 6, 2014.

[12] G. Gariepy et al., "Single-photon sensitive light-in-fight imaging," Nature Communications, vol. 6, p. 6021, 2015.

[13] R. K. Henderson et al., "A $192 \times 128$ time correlated single photon counting imager in 40nm CMOS technology," European Solid-State Circuits Conference, Dresden, pp. 54-57, 2018.

[14] S. Pellegrini et al., "Industrialised single photon avalanche diodes," 2017 IEEE International Electron Devices Meeting (IEDM), pp. 16.5.1-16.5.4, 2017.

[15] P. Alessi et al., "Colorimetry, 3rd edition," Tech. Rep. CIE 015:2004, International Commission on Illumination (CIE), 2004. 